\title{
Antimicrobial Resistance Pattern of Mycobacterium Tuberculosis Complex Isolated from Extrapulmonary Tuberculosis Patientsin Sohag Governorate
}

\author{
MostafaYousef El-Mishad, AbeerSheneef Mohamed, Tamer \\ Mohamed Mahmoud, EkramAbd-El Rahman Mahmoud \\ Medical Microbiology \& Immunology Department, El-Azhar University and Sohag \\ University
}

\begin{abstract}
As the number of drug resistant pulmonary TB is increasing around the world, the number of drug resistant TB with extrapulmonary manifestations are also on rise. However, there is surprisingly scant information in medical literatures on prevalence and impact of extrapulmonary drug-resistant TB

Aim:This study aimed to detect and isolate Mycobacterium Tuberculosis Complex (MTC or MTBC) strains from extrapulmonary samples then determine the drug resistance pattern of these strains to antituberculus drugs.

Materials and Methods: A total of 100 clinical specimens were collected during the study period from September 2016 to January 2018 at Medial Microbiology \& Immunology Department, Sohag Faculty of Medicine from patients suspected to have EPTB. All samples subjected to ZN staining \&cultures on LJ media then all positive culture were subjected for identification of the type of mycobacteria by two methods, phenotypically by biochemical tests and genotypically by conventional PCR fordetection of MTC.Antimicrobial susceptibility pattern was done for MTC strains.
\end{abstract}

Results:Out of 100 collected extrapulmonary samples, 66 samples were positive for mycobacterial culture. By using biochemical tests and PCR for identification of species, 53 isolates were identified as MTC and 13 isolates were identified as NTM. Antimicrobial susceptibility pattern was done for MTCstrains and detected 5 strains were MDR and one strain was XDR.

Conclusion:Extrapulmonary TB infection rate in Sohag governorate is high and the problem of drug resistance in extrapulmonary tuberculosis (EPTB) cannot be overlooked.

\section{INTRODUCTION}

At present, the most serious issue with TB control programme is emergence of multi and extensively drug resistant Mycobacterium tuberculosis strain worldwide (Singh \& Jain, 2015).

Multidrug-resistant tuberculosis (MDR-TB) is defined as tuberculosis caused by bacteria resistant to the two first-line antituberculosis drugs rifampicin and isoniazid (Dheda et al., 2017), While extensively drug resistant tuberculosis( XDR -TB )is defined as TB with resistance to at least isoniazid, rifampin, a fluoroquinolone, and 1 of 3 injectable second-line drugs (amikacin, kanamycin, or capreomycin) (Banerjee et al., 2008).
Several countries, including India, Iran and South Africa, have also reported totally drug-resistant (TDR)-TB strains that are apparently resistant to all tested first-line, second-line and thirdline anti-TB drugs (Dheda et al.., 2017). However, this (TDR-TB) disease entity is currently not endorsed by WHO since drug susceptibility testing (DST) for many secondline/third-line drugs are poorly reproducible (ranging from 50\% to $80 \%$ ), the number of drugs tested varies among reference laboratories and the existing category of XDR-TB already encompasses extensive drug 
resistance to most active anti-TB drugs

2).

Drug resistant-extrapulmonary tuberculosis is considered reason to worry due to following reasons (Singh \& Jain, 2015): (I) Drug resistance in cases of EPTB is increasing and now it cannot be considered as rare. (II) Accurate and timely diagnosis and drug susceptibility testing are very difficult and may result into high morbidity and mortality.(III) DREPTB is often difficult to treat due to poor penetration of some key antitubercular drugs into extra-pulmonary sites (especially in CSF)(IV) HIV and young age are independent key risk factors (v) Although not contagious but it may co-exist with highly contagious pulmonary manifestation. DR-EPTB can arise through acquired-DR-TB from previous improper TB treatment or transmission of DR-strains. A recent metaanalysis demonstrated that when PTB and EPTB coexisted, a higher rate of DR-TB was observed than PTB alone (Pooja et al., 2018).

\section{MATERIALS AND METHODS}

The study was conducted from September 2016 to January 2018 at Medial Microbiology \& Immunology Department, Sohag Faculty of Medicine. The study included patients in different departments at Sohag University Hospital and private clinics at Sohag Governorate who suspected by clinician to have (EPTB).

\section{Specimen collection $\&$ processing}

One hundred clinical specimens were collected during the study period and screened for mycobacteria species. These include 45 urine samples, 35 stool samples and 20 other samples (rather than urine \&stool) include pleural fluid samples (5), ascitic fluid samples (3), biopsies from skin lesions (3), pus from chest abscess (2), cervical lymph nodes biopsy (2), pus from breast abscess (2), biopsy from
(Cegielski et al., 201 laryngeal mass (1), biopsy from vocal cord thickening (1) and swab from skin sinus (1).

Tow smears were prepared from each sample one from the sample directly and another one after decontamination and concentration process. The smears were stained by ZiehlNeelsen stain for detection of acid fast bacilli. The contaminated samples as stool samples were decontaminated by Petroff method. After decontamination of samples sterility test was done for each sample by inoculation on three agar plates (nutrient agar, blood agar and MacConkey) in addition to L.J media and incubate for 3 days if no growth this mean good decontamination but if growth appear on any plate this mean imperfect decontamination and the test repeated again. After that inoculation on Lowenstein Jensen media (LJ) was done with examination once weekly for 12 weeks and record number of days required for colonies to become macroscopically visible and if the pigment was produced or not to differentiate between MTC and NTM

\section{Identification of isolates:}

The isolates from positive mycobacterial culture were identified biochemiclly by sensitivity to inhibitory substances PNB (Para nitro benzoic) acid $t$ and TCH (thiophene-2carboxylic acid hydrazide), nitrate reduction test and niacin test. Mycobacterium tuberculosis complex (MTC) strains were sensitive to PNB, resistant or sensitive for $\mathrm{TCH}$ and had positive niacin \&positive nitrate reduction test. The isolates were further identified by conventional PCR. The used primers were specific for identification of MTC by pair of primers designed to amplify an insertion sequence IS6110. The sequence of these FP1 and RP2 primers were: 5'-CCT GCGAGC GTA 
SOHAG MEDICAL JOURNAL Antimicrobial Resistance Pattern of Mycobacterium Tuberculosis

GGC GTC GG3 and 5' CTC GTC CAGCGC CGC TTC GG 3', respectively).The presence of $123 \mathrm{bp}$ Antimicrobial susceptibility test of MTC: by using proportion method that determined the percentage of growth of a defined inoculum on a drug-free control medium vs. growth on culture fragment indicated a positive test for M. tuberculosis complex.

media containing the critical concentration of an anti-TB drug.

\section{RESULTS}

Out from 100 extrapulmonary samples analyzed, 66 samples were positive for mycobacterial infection. Those positive samples were 46 from male and 20 from female with a mean of age 45.8 .

The overall positivity for mycobacteria observed through $\mathrm{ZN}$ staining and $\mathrm{LJ}$ culture was 47 and 66 respectively. This mean that Sensitivity, specificity, positive predictive value (PPV) and negative predicative value (NPV) of ZN stain method in comparison with L.J Culture media method were $71.21 \%, 100 \%, 100 \%$ and $64.15 \%$ respectively. The incidence of MTC in samples had negative film with positive culture was (16/19), representing $84.2 \%$ while the incidence of MTC in samples had positive film with positive culture was (37/53), representing 69.8\% of total MTC strains in all samples

During our study period, all positive mycobacterialisolates(66) were tested by both biochemical tests and PCR (the gold standard test) for identification of MTC. Fifty six isolates were identified as MTC and 10 isolates as NTM by biochemical tests, whereas 53 isolates were identified as MTC and 13 isolates as NTM by PCR. So sensitivity, specificity, positive predictive value (PPV), negative predicative value (NPV) of biochemical tests in comparison with PCR method were 100\%, 76.9\%, 94.6\% and 100respectively.Table (1) show the incidence of MTB complex among positive samples for mycobacteria in different studied cases.

Table (1): Incidence of MTB complex among positive samples for mycobacteria in different studied cases $($ N. $=66)$

\begin{tabular}{|c|c|c|c|}
\hline \multirow[t]{2}{*}{ Type of sample } & \multicolumn{2}{|c|}{ Positive samples } & \multirow[t]{2}{*}{ Total } \\
\hline & $\begin{array}{r}\text { MTB complex } \\
(\mathrm{N} .=53) \\
\text { NO. }(\%)\end{array}$ & $\begin{array}{c}\text { MOTT } \\
(\text { N.=13) } \\
\text { NO. }(\%)\end{array}$ & \\
\hline Urine samples & $24(88.9 \%)$ & $3(11.1 \%)$ & $27(100 \%)$ \\
\hline Stool samples & $15(68.2 \%)$ & $7(31.8 \%)$ & $22(100 \%)$ \\
\hline \multicolumn{4}{|c|}{ Other extrapulmonary samples } \\
\hline $\begin{array}{lll}\text { Biopsy from } & \text { skin nodule } \\
& \& \& \text { \&ulceration }\end{array}$ & $1(100 \%)$ & $0(0.0 \%)$ & $1(100 \%)$ \\
\hline Swab from skin sinus & $0(0.0 \%)$ & $1(100 \%)$ & $1(100 \%)$ \\
\hline Biopsy from laryngeal mass & $1(100 \%)$ & 0 $(0.0 \%)$ & $1(100 \%)$ \\
\hline Biopsy from vocal cord lesion & $1(100 \%)$ & $0(0.0 \%)$ & $1(100 \%)$ \\
\hline Ascetic fluid & $3(100 \%)$ & $\mathbf{0}(\mathbf{0 . 0 \%})$ & $3(100 \%)$ \\
\hline Pus from chest abscess & $1(100 \%)$ & 0 (0.0\%) & $1(100 \%)$ \\
\hline Plural fluid & $5(100 \%)$ & $\mathbf{0}(\mathbf{0 . 0 \%})$ & $5(100 \%)$ \\
\hline Cervical lymph node biopsy & $2(100 \%)$ & $\mathbf{0}(\mathbf{0 . 0 \%})$ & $2(100 \%)$ \\
\hline Pus from breast abscess & 0 (0.0\%) & $2(100 \%)$ & $2(100 \%)$ \\
\hline
\end{tabular}

Antibiotic susceptibility pattern of MTB complex strains was done for MTC strains as shown in table (2). The incidence of MDR among EPTB cases was $9.4 \%$ while incidwnce of XDR among EPTB cases was 1.9\%.

Table (2): Antibiotic susceptibility pattern of MTB complex strains not done (N. = 53) 
SOHAG MEDICAL JOURNAL Antimicrobial Resistance Pattern of Mycobacterium Tuberculosis

\begin{tabular}{|c|c|c|c|}
\hline \multirow[t]{2}{*}{ Antibiotics } & \multicolumn{2}{|c|}{ Antibiotic susceptibility pattern of MTB complex } & \multirow[t]{2}{*}{ P-value } \\
\hline & $\begin{array}{l}\text { Sensitive } \\
\text { NO. }(\%)\end{array}$ & $\begin{array}{r}\text { Resistant } \\
\text { NO. }(\%)\end{array}$ & \\
\hline \multicolumn{4}{|c|}{ First line antituberculus } \\
\hline Isoniazid & $46(86.8 \%)$ & $7(13.2 \%)$ & 0.111 \\
\hline Rifampicin & $48(90.6 \%)$ & $5(9.4 \%)$ & 0.876 \\
\hline Ethambutol & $52(98.1 \%)$ & $1(1.9 \%)$ & 0.029* \\
\hline Streptomycin & $50(94.3 \%)$ & $3(5.7 \%)$ & 0.279 \\
\hline \multicolumn{4}{|c|}{ Second line antituberculus } \\
\hline Amikacin & $49(92.5 \%)$ & $4(7.5 \%)$ & 0.788 \\
\hline Capreomycin & $51(96.2 \%)$ & $2(3.8 \%)$ & 0.352 \\
\hline Kanamycin & $52(98.1 \%)$ & $1(1.9 \%)$ & $0.029 *$ \\
\hline Ofloxacin & $47(88.7 \%)$ & $6(11.3 \%)$ & 0.197 \\
\hline
\end{tabular}

\section{DISCUSSION}

In our study Mycobacterial infections were more among males $(69.7 \%)$ than females $(30.3 \%)$. This finding was supported by those of Arora and Gupta (2006), Peto et al. (2009) and Hibah study (2015) in El-Behira Egypt.The reported sex-difference in rates of cases with mycobacterial infection may be due to lower notification rate for females. On other hand, some studies showed higher incidence in female more than male these findings showed by Noertjojo et al. (2002), Yang et al. (2004), Forssbohm et al. (2007) and Mohammadien et al. (2017).

In the present study, The age of patients with mycobacterial infection was ranging between 8 years and 80 years with Mean \pm S.D $(45.8 \pm 17.33)$.Our result was in agreement with the following studies which done by Henkle et al. (2017) who reported the median age of patients with extrapulmonary isolateswas 50 years (range $0.8-92$ years), a study by Peto et al. (2009) who reported The mean age of patients with EPTB was 44 years, range (0-105), median(41).On other hand, our results were different from those of Mohammadien et al. (2017) study which done in SohagGovernorate, Egypt from 2010 to 2014 , he reported that the mean agefor extrapulmonary TB is $(34.8 \pm$ 7.1).Also our study was higher than the following studies: a study in Aswan Chest Hospital, Egypt for EPTB cases Mean age \pm SD36.31 \pm 19.53 (Sobh et al., 2016), a study in Australia on EPTB cases Age in years:36 (2080)(Pollett et al., 2016)and a study done at India on 510 different extra pulmonary sampleswith a mean age of 24 years(18, SD) years(Kumari et al., 2016).

In our study Mycobacteria was detected in 66 samples by L.J culture media, whereas Z.N stain detected mycobacteria in only 47 samples. Using the culture as the gold standard test, ZN sensitivity \& specificity reached $71.21 \%$ and $100 \%$ respectivel. Our result was near to $\mathrm{ZN}$ smear sensitivity reported by El-Dawi et al. 2004, which was $65.4 \%$.ZiehlNeelsen stain smear examination has been previously reported to have sensitivity lower than our result. It was $31.3 \%$ (Maurya et al. 2015), $33.79 \%$ (Negi et al.2005) and $41 \%$ (Aderaye et al. 2007). On other hand, some previous result for sensitivity of $\mathrm{ZN}$ microscopic smear examination were higher than our result which ranging from $84 \%$ (WHO) to $88 \%$ (Shinnick and Jonas 1994).

In our studySensitivity, specificity, Positive predicted value (PPV) and negative predicted value (NPV)of biochemical tests in comparison with 
PCR method in identification of mycobacteria specieswere $100 \%$, $76,9 \%, \quad 94.6 \% .100 \%$ respectively. These results disagreed with those results of Sadeghianet al., 2005 who reported that the sensitivity and specificity of biochemicaltests compared to PCR method were 96 and $100 \%$, respectively and Positive predicted value (PPV) and negative predicted value (NPV) were 100 and $92 \%$, respectively.

In our study the incidence of MTC was $53 \%$ from all collected extrapulmonary samples this was higher than another study conducted in India in which the incidence of MTBC was 30\% from all collected extrapulmonary samples (Gupta et al., 2016).

In our study MTC strains were mostly isolated from urine samples ( 24 isolates, representing $45 \%$ of total number of EPTB isolates), followed by stool samples ( 15 isolates, representing $28 \%$ ) then other extrapulmonary samples (14 isolates, representing $26.4 \%, 5$ strains from pleural fluid, 3 strains from ascitic fluid, 2 strains from cervical lymph nodes biopsies, one strain from each biopsy from skin nodule \&ulceration, Biopsy from laryngeal mass, Biopsy from vocal cord lesion \& Pus from chest abscess). This means that renal TB was the commonest site of EPTB followed by TB enteritis, TB pleural effusion, TB ascites and lymphadenitis then equal frequency for each cutaneous TB, laryngeal TB, vocal cord TB and TB in chest wall.This was in accordance with Ranjan and Sharma, (2010) who reported that genitourinary tract (GUT) is the most common site of EPTB. This high percentage of renal TB and TB enteritis may reflect higher rate of suspecion by clinician for renal TB and TB enteritis than previous studies and improvement in diagnostic techniques in our study.
On other hand, it differ from a study in Sohag from 2010 to 2014 by Mohammadien et al. (2017) which depended mainly on histopathological diagnosis (in percent 68\%) but Bacteriological tests were low in percent $(11 \%)$. The sites of EPTB were in TB lymphadenitis (187 cases, $37.4 \%)$, TB pleural effusion (106 cases, $21.2 \%$ ), skeletal TB (90 cases, $18 \%$ ) which included (75cases, $15 \%$ ) that were pott'sdisase and (5 cases, $1 \%$ ) were articular TB (3 cases with knee arthritis, 1 case hip arthritis, and 1 case elbow arthritis) and (10 cases, $2 \%)$ with extraspinal osteomyelitis(7 cases with rib osteomyelitis and 3 cases with femur osteomyelitis), TB ascites (37 cases,7.4\%), TB enteritis (21 cases, $4.2 \%)$, cutaneous TB (10 cases, 2\%), genitourinary TB (30 cases, 6\%) which included (22 cases, $4.4 \%$ ) renal TB and (4 cases, 0.8 ) were male genital TB cases ( 3 cases TB epididymitis and 1 case testicular TB)and (4 cases, $0.8 \%$ ) were female genital TB cases ( 3 cases TB salpingitis, 1 case $\mathrm{TB}$ endometritis), CNS TB (10 cases, 2\%) which included (7 cases, $1.4 \%$ ) were TB meningitis and (3 cases, $0.6 \%$ ) were TB tuberculoma, pericardial effusion ( 5 cases, 1\%), TB mastitis (3 cases, $0.6 \%$ ), TB laryngitis (1 case, $0.2 \%$ ). Also at Aswan Chest Hospital, the most common affected extrapulmonary TB site was lymph nodes followed by pleura, and then bone $(27.4 \%, 25 \%$, and 14.9\%, respectively (Sobh et al., 2016). It differ also from Hibah study (2015) in El-Behira Governorate, Egypt, who reportedPleural TB constituted $63.3 \%(n=1341)$ of all EPTB cases followed by Lymph node TB constituted 20\% $(n=427)$ of EPTB cases. Bone TB constituted $7.5 \%(n=$ 157) cases, genital TB constituted $3.3 \%(n=70)$ of all EPTB cases, Renal TB constituted $1.6 \%(n=34)$ of allEPTB cases. Also differ from Peto 
SOHAG MEDICAL JOURNAL Antimicrobial Resistance Pattern of Mycobacterium Tuberculosis

et al (2009) who reported extrapulmonary tuberculosis sites of disease in United States at 1993-2006 ( $\mathrm{N}=47,293)$ including lymphatic (40.4\%), pleural (19.8\%), bone and/or joint $(11.3 \%)$, genitourinary $(6.5 \%)$, meningeal $(5.4 \%)$, peritoneal $(4.9 \%)$, and unclassified EPTB $(11.8 \%)$ cases. These differences suggest that the dynamics of extrapulmonary tuberculosis epidemiology may be specific to geographic location and population and also affected by method of diagnosis and length of period during which the samples collected.

In our study the incidence of NTM represented $13 \%$ from total collected extrapulmonary specimen. It was in accordance with a study from a Northern Indian population in which $18.2 \%$ of the NTM were recovered fromextrapulmonary specimens (Umrao et al., 2016). And also our result was near to Chakrabarti et al. (1990) and Das et al. (1982) they reported incidence of NTM from different regions of India were $7.4 \%$ and $8.3 \%$ respectively. In our study NTM strains were mostly isolated from stool samples ( 7 isolates, representing $53.8 \%$ of total number of NTM isolates), followed by urine samples (representing 23\%), Pus from breast abscess (representing 15\%), then Swab from skin sinus (representing 7.6\%). This was different from a study by Henkle et al. (2017) in Oregon, USA, during 2007-2012. Among the 334 extrapulmonary NTM infections, 197 $(59.0 \%)$ were skin/soft tissue, 57 $(17.1 \%)$ were disseminated, $28(8.4 \%)$ were lymph node, 14 (4.2\%) were joint, and $38(11.4 \%)$ were other. In another study, Most isolates of NTM obtained from extrapulmonary samples were from urine, followed by exudate/abscess, skin biopsy, lymph node biopsy, and stool.(represented $54 \%, 16.6 \%, 11.1 \%, 9.7 \%, 8.3 \%$ respectively of total number of extrapulmonary isolated NTM.

(González et al., 2017).

In the present study out of 53 positive MTC cases, 37 samples were found to be positive by $\mathrm{ZN}$ staining (representing 69.8\%) this result was near to result of Gupta et al. (2016) that found out of 30 positive MTBC cases from extrapulmonary samples, 18 samples were positive by $\mathrm{ZN}$ staining (representing 60\%). These results are concordant with various studies done by Githui et al. 1993 found $(65 \%$ by $\mathrm{ZN}$ staining), Ulukanligil et al. 2000 found (67.6\% by $\mathrm{ZN}$ staining), Murray et al. 2003 found $(73 \%$ by $\mathrm{ZN}$ staining), Prashanthi et al. 2005 found $(50 \%$ by ZN staining) but our result was higher than Jain et al. 2002 found (32\% positive by $\mathrm{ZN}$ staining).In spite of the high specificity of ZN staining method, it showed variable and low sensitivity which is mainly attributed to the degree of mycobacterium shedding in a sample.

Diagnosis extra-pulmonary tuberculosis (EPTB) in smear-negative patients can be difficult. In our study total number of positive culture for MTBC from total extrapulmonary samples were 53. The incidence of positive film in samples had positive culture for MTBC was 37/53 representing $69.8 \%$ of total MTBC strains while incidence of negative film in samples had positive culture for MTBC was(16/53), representing $30.2 \%$ of total MTBC strains. This result was differing from a study performed at Italy, In which the total number of positive culture for MTC from extrapulmonary samples were 112. The incidence of positive film in samples had positive culture for MTBC was 15/112 (representing $13.4 \%$ ), while incidence of negative film in samples had positive culture for MTBC was 97/112 ( $86.6 \%$ ), in this study sensitivity of microscopy was 
poor probably due to Ziehl-Neelsen staining before sample concentration but in our study concentration of samples before staining was done and this raise the sensitivity of $\mathrm{ZN}$ smear (

Lombardil et al., 2017).

Antibiotic susceptibility pattern of isolated MTBC strains in our study showed that the strains had the highest sensitivity rates to both Ethambutol and Kanamycin (each represented 98.1\%), Capreomycin( 96.2\%), Streptomycin $(94.3 \%), \quad$ Amikacin $(92.5 \%)$ then Rifampicin( $90.6 \%)$. Meanwhile, the highest resistance rates were to Isoniazid (13.2\%) ,Ofloxacin(11.3\%), Rifampicin(9.4\%), Amikacin(7.5\%). In another study by Nair et al. (2009) showed resistance pattern of MTBC extrapulmonary towards first and second line drugs by LJ proportion method, it supported our result that the strains had highest resistance to isoniazid 16/54 (29.6\%) and highest sensitivity to ethambutol but it differ from our result in the following resistance after isoniazid. It followed by rifampcin 8/54 (14.8\%), pyrazinamide 7/54(12.9\%), ethionamide, kanamycin and capreomycin (each 6/54represented $11.1 \%)$ then ciprofloxacin and amikacin(each 5/54 represented 9.2\%) then ethambutol 4/54(7.4\%).Also Pollett et al. (2016) reporteddrugsusceptibility testing results (by phenotypic or genotypic methods) which were lower than our result. There were $: 5 \%(3 / 66)$ had isoniazid resistance, $2 \% \quad(1 / 66)$ had pyrazinamide resistance, $2 \% \quad(1 / 66)$ had rifampicin resistance.

In our result drug resistance for the first-line antituberculos drugs were: Isoniazid (13.2\%), rifampicin ( $9.4 \%$ ), Ethambutol (1.9\%), Streptomycin (5.7\%), while in a study done in the Netherlands from 1998 to 2005 and amounted the levels of drug resistance for the first-line antituberculosis drugs
$7.5 \%$ for isoniazid, $1.4 \%$ for rifampin, $8.5 \%$ for streptomycin, and $1.0 \%$ for ethambutol(van et al., 2007). Alsoisoniazid resistance was lower in another study by Kumariet al. (2016) who reported INH mono resistance in $1 / 51 \quad(1.96 \%)$ ).In another study isoniazid monoresistance was $(9.9 \%)$. rifampicin monoresistance was $(2.1 \%)$, streptomycin monoresistance was $(0.5 \%)$, and ethambutolmonoresistancewas $(0.9 \%)$. prevalence of ofloxacinresistance i.e. pre-XDR.TB among MDR-TB patients was $17 \%$. ( Sharma et al., 2017). Also in a study by Kohli et al. (2016), DST by proportion method showed near result to our study in the incidence of rifampcin resistance $6.7 \%$, isoniazid resistance $14.7 \%$, but had higher result in incidence of streptomycin resistance $22.7 \%$, ethambutol resistance 9.3\%.

In our study MDR represented $9.4 \%$ of total MTBC isolated strains. Though very few data is available on drug resistance in extra pulmonary tuberculosisour finding is supported by data from previous studies which reported $12.5 \%$ MDR- EPTB in Nepal and $10 \%$ in Delhi India (Gurung et al., 2010) (Sachdeva et al., 2002). Also our result was higher than those reported by Qian et al. (2018) who identified Multidrug-resistant TB (MDR-TB)in5/1259( 0.4\%)and Pollett et al. (2016) who reportednone had multi-drug resistance while, our result was lower than that reported by Kumari et al. (2016) who identified MDR-TB in $14 / 51 \quad(27.45 \%)$. In another study, prevalence of MDR-TB was $3 \%$ in treatment - naive \& $14.5 \%$ in treatment -experienced EPTBcases(Sharma et al., 2017).

In our study XDR: represented $1.9 \%$ of total MTBC isolated strains these results in agreement with that of Sharma et al. (2017) who found prevalence of extensively-drug resistant tuberculosis (XDR-TB) 
among MDR in Extrapulmonary Tuberculosis Cases was 2.6 but disagreed with those of Balaji et al. (2010) who reported the rare possibility for the presence of XDR strain in extraplumonary site in India and Qian et al. (2018) who identified no XDR in EPTB cases. Also our result about XDR was lower than that of Nene et al. (2016)who reported XDR among EPTB cases 2/29 (representing 6.9\%).

Our findings are also supported by data accrued from different countries that found the prevalence of MDR may lie between 1\%-69\% of total of EPTB cases; whereas, the proportion of resistant cases to any one antituberculosis drug is about 10\%-75\%. The wide variation in proportion of drug resistant EPTB among different studies is probably due to variation in study settings, burden of MDR-TB and quality of medical services in particular region, demographic characteristic and HIV status of patients, types of EPTB cases investigated, sample size and its selection criteria etc (Singh \& Jain, 2015).

In conclusion, we got a significant number of MTC isolated from extra pulmonary cases of our Governorate which suggests that this form of TB has also reached an alarming level so this uncommon form of tuberculosis cannot be overlooked and due attention on the patients of extra pulmonary tuberculosis should be given. Antibiotic susceptibility pattern should be done for each of isolated MTC strains to optimize efficient anti-TB treatment and to avoid development of MDR- TB which ultimately develop to XDR-TB.

\section{REFERENCES}

1- Aderaye, G., Aseffa, A., Worku, A., \& Lindquist, L. (2007).Comparison of acid-fast stain and culture for Mycobacterium tuberculosis in pre-and post-bronchoscopy sputum and bronchoalveolar lavage in HIV-infected patients with atypical chest $\mathrm{X}$-ray in Ethiopia. Annals of thoracic medicine, 2(4), 154-157.

2- Arora, V. K., \& Gupta, R. (2006). Trends of extra-pulmonary tuberculosis under revised national tuberculosis control programme: A study from South Delhi. Indian Journal of Tuberculosis, 53(2), 77.

3- Balaji, V., Daley, P., Anand, A. A., Sudarsanam, T., Michael, J. S., Sahni, R. D., ...\& John, K. R. (2010). Risk factors for MDR and XDR-TB in a tertiary referral hospital in India. PloS one, 5(3), e9527.

4- Banerjee, R., Allen, J., Westenhouse, J., Oh, P., Elms, W., Desmond, E., ...\& Flood, J. (2008). Extensively drugresistant tuberculosis in California, 1993-2006. Clinical Infectious Diseases, 47(4), 450-457.

5- Cegielski, P., Nunn, P., Kurbatova, E. V., Weyer, K., Dalton, T. L., Wares, D. $F$., $\quad$...\&Raviglione, $M$. (2012). Challenges and controversies in defining totally drug-resistant tuberculosis. Emerging infectious diseases, 18(11), e2.

6- Chakrabarti, A., Sharma, M., \&Dubey, M. L. (1990). Isolation rates of different mycobacterial species from Chandigarh (north India). The Indian journal of medical research, 91, 111114.

7- 7- Das, B. K., Sharma, V. K., Bhau, L. N., Saxena, S. N., \&Bhardwaj, B. K. (1982). Characterisation of mycobacterial strains from clinical specimens. Indian journal of pathology \& microbiology, 25(1), 19.

8- Dheda, K., Gumbo, T., Maartens, G., Dooley, K. E., McNerney, R., Murray, M., ...\&Theron, G. (2017).The epidemiology, pathogenesis, transmission, diagnosis, and management of multidrug-resistant, extensively drug-resistant, and incurable tuberculosis. The lancet Respiratory medicine, 5(4), 291-360.

9- El-Dawi, T. G., Saeed, N. S. E. N., \& Hamid, M. E. (2004). Evaluation of a PCR-amplified IS6110 insertion element in the rapid diagnosis of 
pulmonary tuberculosis in comparison

to microscopic methods in

Sudan. Saudi medical journal, 25(11), 1644-1647.

10-Forssbohm, M., Zwahlen, M., Loddenkemper, R., \&Rieder, H. L. (2007).Demographic characteristics of patients with extrapulmonary tuberculosis in Germany. European respiratory journal, 31:99-105.

11- Githui, W., Kitui, F., Juma, E. S., Obwana, D. O., Mwai, J., \&Kwamanga, D. (1993). A comparative study on the reliability of the fluorescence microscopy and ZiehlNeelsen method in the diagnosis of pulmonary tuberculosis. East African medical journal, 70(5), 263-266.

12- González, S. M., Cortés, A. C., Yoldi, L. A. S., García, J. M. G., Álvarez, L. M. A., \& Gutiérrez, J. J. P. (2017). Micobacterias no tuberculosas. $i$ Unaamenazaemergente?. Archivos de Bronconeumología, 53(10), 554-560.

13- Gupta, S., Goyal, R. K., Bareja, R., \&Behara, R. N. (2016). Evaluation of various culture and staining techniques for the detection of extra pulmonary tuberculosis. International Journal of Research in Medical Sciences, 4(9), 3982-3987.

14- Gupta, S., Goyal, R. K., Bareja, R., \&Behara, R. N. (2016). Evaluation of various culture and staining techniques for the detection of extra pulmonary tuberculosis. International Journal of Research in Medical Sciences, 4(9), 3982-3987.

15- Gurung, R., Bhattacharya, S. K., Pradhan, B., Gurung, S., \& Singh, Y. I. (2010). Phenotypic characterisation and drug sensitivity testing of mycobacteria isolated from extrapulmonary tuberculosis. Kathmandu University Medical Journal,8(1), 5761.

16- Henkle, E., Hedberg, K., Schafer, S. D., \& Winthrop, $K$. L. (2017).Surveillance of ExtrapulmonaryNontuberculous Mycobacteria Infections, Oregon, USA, 2007-2012. Emerging infectious diseases, 23(10), 1627.
17- Hibah, N. A. A. (2015).Extrapulmonary tuberculosis situation in El-Behira Governorate, Egypt. Egyptian Journal of Bronchology, 9(1), 96-100.

18- Jain, A., Bhargava, A., \&Agarwal, $S$. K. (2002). A comparative study of two commonly used staining techniques for acid fast bacilli in clinical specimens. Indian Journal of Tuberculosis, 49(3), 161-162.

19- Kohli, A., Bashir, G., Fatima, A., Jan, A., \& Ahmad, J. (2016). Rapid drug-susceptibility testing of Mycobacterium tuberculosis clinical isolates to first-line antitubercular drugs by nitrate reductase assay: A comparison with proportion method. International journal of mycobacteriology, 5(4), 469-474.

20- Kumari, R., Sinha, P., Banerjee, T., \&Anupurba, S. (2016). Isolation and characterization of Mycobacterium tuberculosis from extra pulmonary specimens in a tertiary referral hospital of north India.Journal of Advances in Medicine, 5(2), 25-28.

21- Lombardi, G., Di Gregori, V., Girometti, N., Tadolini, M., Bisognin, F., \& Dal Monte, P. (2017). Diagnosis of smear-negative tuberculosis is greatly improved by Xpert MTB/RIF. PloS one, 12(4), e0176186.

22- Maurya, A. K., Nag, V. L., Kant, S., Kushwaha, R. A. S., Kumar, M., Singh, A. K., \& Dhole, T. N. (2015). Prevalence of nontuberculous mycobacteria among extrapulmonary tuberculosis cases in tertiary care centers in Northern India. BioMed research international, 2015.

23- Mohammadien, H., Alkhayat, K., Hamed, A., \&Shaaban, M. (2017).Patterns, trends and treatment outcomes of extra-pulmonary tuberculosis in Sohag, Upper Egypt. Egyptian Journal of Chest Diseases and Tuberculosis, 66(2), 313316.

24- Murray, S. J., Barrett, A., Magee, J. G., \& Freeman, $R$. (2003).Optimisation of acid fast smears for the direct detection of mycobacteria 
in clinical samples. Journal of Clinical Pathology, 56(8), 613-615.

25- Nair, D., Capoor, M. R., Rawat, D., Srivastava, L., \&Aggarwal, P. (2009). Standardization of first and second-line antitubercular susceptibility testing using BacT Alert 3D system: a report from a tertiary care centre in India. Brazilian Journal of Infectious Diseases, 13(6), 422-426.

26- Negi, S. S., Khan, S. F., Gupta, S., Pasha, S. T., Khare, S., \&Lal, S. (2005). Comparison of the conventional diagnostic modalities, bactec culture and polymerase chain reaction test for diagnosis of tuberculosis. Indian journal of medical microbiology, 23(1), 29.

27-Nene, A., Kewalramani, N., \&Agarwal, $V$. (2016). Prospective study analysing prevalence of XDR TB in Mumbai, India.

28- Noertjojo, K., Tam, C., Chan, S., \& Chan-Yeung, M. M. (2002). Extrapulmonary and pulmonary tuberculosis in Hong Kong. The International Journal of Tuberculosis and Lung Disease, 6(10), 879-886.

29- Peto, H. M., Pratt, R. H., Harrington, T. A., LoBue, P. A., \& Armstrong, L. R. (2009). Epidemiology of extrapulmonary tuberculosis in the United States, 1993-2006. Clinical Infectious Diseases, 49(9), 1350-1357.

30- Pollett, S., Banner, P., O'Sullivan, M. V., \& Ralph, A. P. (2016). Epidemiology, diagnosis and management of extra-pulmonary tuberculosis in a low-prevalence country: a four year retrospective study in an Australian Tertiary Infectious Diseases Unit. PloS one, 11(3), e0149372.

31- Pollett, S., Banner, P., O'Sullivan, M. V., \& Ralph, A. P. (2016). Epidemiology, diagnosis and management of extra-pulmonary tuberculosis in a low-prevalence country: a four year retrospective study in an Australian Tertiary Infectious Diseases Unit. PloS one, 11(3), e0149372.

32- Pollett, S., Banner, P., O'Sullivan, M. V., \& Ralph, A. P. (2016).
Epidemiology, diagnosis and management of extra-pulmonary tuberculosis in a low-prevalence country: a four year retrospective study in an Australian Tertiary Infectious Diseases Unit. PloS one, 11(3), e0149372.

33- Pooja S., Surya K., Priyanka G., Abhilasha T., Sarika P.( 2018):Extra Pulmonary Tuberculosis: An Overview and Review of Literature, Int. J. Life. Sci. Scienti. Res, January 2018.

34- Prasanthi, K., \&Kumari, A. R. (2005).Efficacy of fluorochrome stain in the diagnosis of pulmonary tuberculosis co-infected with HIV. Indian Journal of Medical Microbiology, 23(3), 179.

45-Qian, X., Nguyen, D. T., Lyu, J., Albers, A. E., Bi, X., \&Graviss, E. A. (2018).Risk factors for extrapulmonary dissemination of tuberculosis and associated mortality during treatment for extrapulmonary tuberculosis. Emerging microbes \& infections, 7(1), 102.

35- Ranjan, K. P., \& Sharma, M. (2010).An approach to the detection of mycobacteria in clinically suspected cases of urinary tract infection in immunocompromised patients.

36- Sachdeva, R., Gadre, D. V., \&Talwar, V. (2002). Characterisation\& drug susceptibility patterns of extrapulmonary mycobacterial isolates. Indian Journal of Medical Research, 115, 102.

37- Sadeghian,A., Tavakkol A., Ghanaat J., Sadeghain H., Namaii M. and Khodadoost M.(2005). Comparison of Three Methods for the Identification of Mycobacterium tuberculosis, INTERNATIONAL JOURNAL OF AGRICULTURE \& BIOLOGY15608530/07-2-203-206

38- Sharma, S. K., Chaubey, J., Singh, B. K., Sharma, R., \& Mittal, A. (2017). Drug Resistance Pattern AmongExtrapulmonary Tuberculosis Cases In A Tertiary Care Center In India. In A61. TUBERCULOSIS DISEASE: HOST RESPONSE AND DIAGNOSTICS (pp. A2086-A2086). American Thoracic Society. 
39- Shinnick T, Jonas V (1994). Molecular approaches to diagnosis of tuberculosis. In: Bloom BR, editors. Tuberculosis. Pathogenesis, Protection and Control, ASM Press, Washington (DC), 517-530.

40- Singh, P. K., \& Jain, A. (2015).Epidemiological perspective of drug resistant extrapulmonary tuberculosis. World J Clin Infect Dis, 5(4), 77-85.

41- Sobh, E., Kinawy, S. A. E., Abdelkarim, Y. M. A., \&Arafa, M. A. (2016). The pattern of tuberculosis in Aswan Chest Hospital, Egypt. International journal of mycobacteriology, 5(3), 333-340.

42- Ulukanligil, M., Aslan, G., \&Tasçi, $S$. (2000). A comparative study on the different staining methods and number of specimens for the detection of acid fast bacilli. Memorias do InstitutoOswaldo Cruz, 95(6), 855-858.
44- Umrao, J., Singh, D., Zia, A., Saxena, S., Sarsaiya, S., Singh, S., ...\& Dhole, T. N. (2016). Prevalence and species spectrum of both pulmonary and extrapulmonarynontuberculous mycobacteria isolates at a tertiary care center. International journal of mycobacteriology, 5(3), 288-293.

45- Van B. K., Mirjam D., Tridiav.L., Kristin K., and Dick van Soolingen. (2007). Drug Susceptibility Testing of Mycobacterium tuberculosis Complex by Use of a High-Throughput, Reproducible, Absolute Concentration Method, JOURNAL OF CLINICAL MICROBIOLOGY, Vol. 45, No. 8, p. 2662-2668.

46- Yang, Z., Kong, Y., Wilson, $F$., Foxman, B., Fowler, A. H., Marrs, C. $F$., ...\& Bates, $J . \quad H$. (2004).Identification of risk factors for extrapulmonary tuberculosis. Clinical infectious diseases, 38(2), 199-205. 
SOHAG MEDICAL JOURNAL Antimicrobial Resistance Pattern of Mycobacterium Tuberculosis 